\title{
Consumo crónico de benzodiazepinas y riesgo de deterioro cognitivo en el anciano
}

Long Term Benzodiazepines Use and Cognitive Decline in the Elderly:The Epidemiology of Vascular Aging Study. Paterniti, Sabrina MD, Dufouil y col.Journal of Clinical Psychopharmacology.2002 Jun; 22:285-293.

\section{Objetivo}

Evaluar si el consumo crónico de benzodiazepinas se asocia con un incremento en la velocidad de deterioro cognitivo en la ancianidad.

Diseño

Estudio poblacional de cohorte prospectivo con seguimiento a 2 y 4 años.

Lugar

Ciudad de Nantes, Francia.

\section{Participantes}

1.389 voluntarios entre 60 y 70 años. Se excluyeron aquellos que hubieran permanecido por tiempo prolongado en instituciones geriátricas.

\section{Evaluación de factores de riesgo}

Datos sobre nivel educacional (años de escolaridad), ocupación, hábitos personales (tabaquismo, consumo de alcohol) y medicamentos fueron recabados a través de entrevistas personales. Se utilizaron el CES-D (versión francesa de la escala de depresión del centro de estudio epidemiológico) y el tratado de Spielberger para evaluar síntomas de depresión y ansiedad respectivamente. Enfermedad coronaria, hipertensión, diabetes y dislipemia fueron considerados potenciales confundidores.

\section{Medición de resultados principales}

Los participantes fueron clasificados como no consumidores, consumidores esporádicos, recurrentes o crónicos de benzodiazepinas según reportaran su uso en una, dos o tres de las evaluaciones realizadas.

El Mini Mental Test se utilizó para evaluar estado cognitivo global; El Trail Making Test, parte B (TMT-B) atención visual y velocidad de ejecución motriz; el Digit Symbol Substitution (DSS) razonamiento lógico, velocidad psicomotriz y atención; Auditory Verbal Learning Test (AVLT) memoria verbal y el Finger Tapping Test (FTT) velocidad de ejecución psicomotriz.

\section{Resultados principales}

Los participantes que consumían crónicamente benzodiazepinas presentaron un incremento significativo del riesgo en el deterioro cognitivo, evaluado a través del Mini Mental Test: odds ratio (OR) [95\% de intervalo de confianza $(\mathrm{Cl})]=1.9$ [1.0-3.5]; DSS: OR [95\% $\mathrm{Cl}]=2.7$ [1.6-4.7]; TMT-B: OR [95\% Cl] $=2.1$ [1.2-3.7].

Si bien los consumidores esporádicos y recurrentes presentaban scores más bajos que los no consumidores, esta diferencia no fue estadísticamente significativa. Los resultados fueron independientes de la edad, sexo, nivel educacional, consumo de tabaco, alcohol y otras drogas psicotrópicas, ansiedad y depresión.

\section{Conclusión}

El consumo crónico de benzodiazepinas aumenta el riesgo de deterioro cognitivo progresivo en la ancianidad.

\section{Comentario}

Desde la aparición de la drogas tranquilizantes a mediados de la década del 50, las mismas se han convertido en una herramienta ampliamente utilizada en la práctica asistencial. De hecho, estadísticas recabadas en EEUU muestran que las benzodiazepinas representan aproximadamente el $17 \%$ del total de drogas prescriptas por año y que $85 \%$ de los sujetos que las consumen jamás han visitado a un psiquiatra en sus vidas.

Esto ha generado sin duda un problema de salud y un motivo de preocupación general que lo ha llevado al ámbito de la investigación. Numerosos estudios se han realizado a fin de documentar los efectos adversos de las benzodiazepinas sobre la atención, memoria y performance psicomotriz. Muchos de ellos con errores metodológicos que limitaron sus alcances.

El estudio EVA con una cohorte poblacional numerosa seguida longitudinalmente sustenta sus resultados, con poder estadístico, sobre la base de dos de los cinco test utilizados (DSS y TMT-B). El hecho de haber seleccionado una cohorte con características educacionales y socioeconómicas superior a la media de la población general, excluyendo posteriormente en un sub-análisis aquellos con puntaje menor a 24 en el Mini Mental Test, fortalece los hallazgos, intentado eliminar el sesgo de una muestra con probabilidades de alojar sujetos con demencia. Si bien alcanzar dicha certeza, resulta imposible.

Vale destacar ciertas limitaciones metodológicas, algunas comentadas por los autores en la discusión. La primera es que existe evidencia sobre efectos colaterales de las benzodiazepinas en relación a la dosis administrada y su vida media. Este estudio no cuenta con información sobre este tipo de datos.

En segundo lugar no hay una medida objetiva para medir la exposición, como los niveles plasmáticos de la droga;tampoco del tiempo transcurrido entre la toma de la misma y la ejecución del test cognitivo, por lo cual resulta difícil concluir si los resultados se deben a la asociación entre dicho deterioro y el consumo crónico o a un efecto secundario agudo de la droga.

Si bien se identificaron algunos factores de riesgo como potenciales confundidores, otros como historia psicopatológica (se desconoce por ej. si las benzodiazepinas fueron prescriptas por algún trastorno específico de ansiedad) y otras comorbilidades no fueron tomadas en cuenta en el análisis.

Conclusión del comentador: Por todo lo expuesto, se podría concluir que si bien los individuos que consumen crónicamente benzodiazepinas presentan más riesgo de sufrir deterioro cognitivo progresivo que los no consumidores, el siguiente trabajo no es contundente en cuanto a determinar asociación causal, siendo sin embargo sus hallazgos, un paso más en el camino de la investigación en este terreno.

\section{Dra. Cecilia Maria Irurzun [ Fellow de clínica médica, Centro Medicus ]}

\section{Bibliografía}

1. Catalan J, Gath D H. Benzodiazepines in general practice: time for a decision. Review. British Medical

Journal 1985 May 11;290:1374-76

2. Catalan J, Gath D, Edmonds G. The Effects of Non-Prescribing of Anxiolytics in general Practice. British Journal of psychiatry 1984; 144:593-602.

3. Hugh J, Mitchell B, Glen D.National Patterns of Psychotherapeutic Drug Use.Arch Gen Psychiatryc 1973 Jun;28:769-83. 